Check for updates

Cite this: RSC Adv., 2017, 7, 28754

\title{
Synthesis of short-chain passivated carbon quantum dots as the light emitting layer towards electroluminescence
}

\author{
Yuanfei Ding, ${ }^{\text {ab }}$ Feng Zhang, ${ }^{\text {ab }}$ Jiacong $X u^{a b}$ Yanqin Miao, ${ }^{\text {ab }}$ Yongzhen Yang, ${ }^{\text {*ab }}$ \\ Xuguang Liu (D) *ac and Bingshe X $u^{\mathrm{ab}}$
}

\begin{abstract}
Short-chain passivated fluorescent carbon quantum dots (S-CQDs) with graphitic structure were synthesized via a one-step hydrothermal approach using phthalic acid with a benzene ring conjugated structure as the carbon source and ethylenediamine as a nitrogenous additive. The synthesized S-CQDs have an average diameter of $5.53 \mathrm{~nm}$ and exhibit excitation-dependent photoluminescence emission. The S-CQD aqueous solution emits bright blue light under ultraviolet excitation with a quantum yield of $29.3 \%$. The optical band gap of the S-CQDs was calculated to be $4.26 \mathrm{eV}$, the highest occupied molecular orbital and the lowest unoccupied molecular orbital energy levels of -8.35 and $-4.09 \mathrm{eV}$ were obtained for the S-CQDs by cyclic voltammetry. In addition, the S-CQDs have a good film-forming ability, as confirmed by atomic force microscopy analysis. By virtue of the structure characteristics, optical properties and the filmforming ability of S-CQDs, quantum dot-based electroluminescent light emitting diodes (QD-LEDs) were fabricated by a solution processing method with S-CQDs as the emitting layer, which emits blue light at 6-9 V driving voltage, showing that the S-CQDs have promising prospects for application in QD-LEDs.
\end{abstract}

Received 27th February 2017

Accepted 24th May 2017

DOI: 10.1039/c7ra02421e

rsc.li/rsc-advances
Carbon quantum dots (CQDs) ${ }^{18}$ are one new class of fluorescent QDs. Serving as optical materials, CQDs possess not only optical properties of semiconductor QDs (e.g., tunable luminescence emission and high photostability) but also particular merits including low toxicity, low cost, chemical stability and environmental friendliness. ${ }^{\mathbf{1 9 , 2 0}}$ Now, regarding the application of optoelectronic devices, CQDs mainly exert their photoluminescence (PL) properties as light conversion phosphors for white light emitting diodes (LEDs). ${ }^{21,22}$ Meanwhile, researchers have gradually been exploring the electroluminescent (EL) properties of CQDs as emitting layer to develop carbon quantum dot-based electroluminescent light emitting diodes (CQD-LEDs). The electroluminescent properties of CQDs allow electrons and holes to form excitons in CQDs, and the excitons decay to stimulate the emission of CQDs. ${ }^{15,23-25}$ However, the investigations about the new members as emitting layer of the electroluminescent devices are only in the initial stage. Zhang et al. prepared a blue emitting CQD-LEDs with a turn-on voltage of $5 \mathrm{~V}$ and a maximum brightness of $24 \mathrm{~cd} \mathrm{~m}^{-2}$ using CQDs with fluorescence quantum yield (QY) of $\approx 40 \%$ as emitting layer that were synthesized by thermally carbonizing citric acid in hot octadecene with 1-hexadecylamine as the passivation agent; ${ }^{25}$ Similarly, Veca et al. fabricated a bluish white emitting CQDLEDs with a maximum brightness of $0.14 \mathrm{~cd} \mathrm{~m}^{-2}$ taking graphitic structure CQDs as emitting layer that were prepared by oxidizing carbon nano-powder sample in aqueous nitric acid solution and then amidating the sample with $\mathrm{PEG}_{1500 \mathrm{~N}}$ in $\mathrm{SOCl}_{2}$ medium. ${ }^{23}$ Wherein, the graphitic structure of the CQDs is 
conducive to electron transfer. Moreover, the two kinds of CQDs used by Zhang et al. ${ }^{25}$ and Veca et $a .^{23}$ have the long-chain passivation layer. The presence of surface passivating agent can improve the optical properties of CQDs. ${ }^{18}$ However, the long-chain passivating endows the surface of CQDs with the organic ligand insulating layer in a thickness of larger than $0.5 \mathrm{~nm}$, which induces a minimal tunnelling barrier to hinder the injection of electrons and holes. Compared with the longchain passivated CQDs, the short-chain passivated CQDs have no insulating layer of larger than $0.5 \mathrm{~nm}$ thickness on their surfaces. The absence of the minimal tunneling barrier reduces the obstacle of carrier injection, thus improving the carrier injection ability. ${ }^{6}$ In other words, with respect to the long chains, the short-chain cap on the CQDs shortens the distances of carriers from CQDs, which probably facilitates the injection of carriers into CQDs more easily. ${ }^{26,27}$ Hence, it is necessary to find a simple and effective method for synthesizing CQDs with good carrier injection mobility and superior QY, and then, as light-emitting layers to achieve CQD-LEDs with high performance.

In this paper, ethylenediamine (EDA) was used as nitrogenous short-chain additive to improve the carrier injection ability of CQDs and to obtain superior QY. Meanwhile, phthalic acid (PA) with benzene ring conjugated structure was used as carbon source for preparing the short-chain passivated fluorescent carbon quantum dots (S-CQDs) with high electrical conductivity and graphitic structure. A one-step hydrothermal method was chosen to simply and effectively prepare S-CQDs. Then, the SCQDs were dispersed in toluene solvent as emitting layer for the fabrication of CQD-LEDs. A 40 nm-thick poly (ethylenedioxythiophene)polystyrene sulfonate (PEDOT:PSS) film as hole injection layer (HIL) and a $40 \mathrm{~nm}$-thick 1,3,5-tris $(\mathrm{N}$ phenylbenzimidazol-2-yl)benzene (TPBI) film as electron transport layer (ETL) were inserted into the two sides of the emitting layer to improve the injection efficiency of holes from ITO cathode and electrons from $\mathrm{LiF} / \mathrm{Al}$ anode, which follows the work by Wang et al. ${ }^{15}$ This method was used to fabricate CQDLEDs with improved performance. Schematic illustration of synthesizing S-CQDs for CQD-LEDs is shown in Scheme 1.

\section{Experimental}

\section{Materials}

PA was obtained from Guangfu Technology Development Co., Ltd (Tianjin, China). EDA was purchased from Fuchen Chemical Reagent Factory (Tianjin, China). All chemicals were commercially available and were used without further purification.

\section{Synthesis of CQDS}

S-CQDs were prepared by one-step hydrothermal treatment using PA (1.66 g, $0.01 \mathrm{~mol}$ ) as carbon source, EDA (0.67 mL, 0.01 $\mathrm{mol})$ as additive and deionized water $(40 \mathrm{~mL})$ as solvent. Typically, PA, EDA and deionized water were shifted into a $100 \mathrm{~mL}$ Teflon-lined stainless-steel autoclave and heated at $200{ }^{\circ} \mathrm{C}$ for $3 \mathrm{~h}$. After cooling to room temperature, the crude solution was filtered with a $0.22 \mu \mathrm{m}$ polyethersulfone membrane to remove larger nanoparticles. Then the resulting S-CQDs solution was further purified in a dialysis bag (cutoff molecular weight: 1000 $\mathrm{Da}$ ) for $12 \mathrm{~h}$ to remove impurities and the water was changed every $3 \mathrm{~h}$. After these steps, the S-CQDs aqueous solution was collected for characterization such as ultraviolet-visible absorption, emission behavior, and QY measurements. Then the powder S-CQDs were acquired by freeze drying and kept for characterization of morphology, chemical composition, electrochemical property or redispersed in toluene to prepare $S$ CQDs toluene solution ( $1 \mathrm{mg} \mathrm{mL}^{-1}$ ) by ultrasonic dispersion for further use. Long-chain passivated fluorescent carbon quantum dots (L-CQDs) were prepared by one-step solvothermal treatment using PA $(0.83 \mathrm{~g}, 0.005 \mathrm{~mol})$ as carbon source, 1-hexadecylamine $(1.21 \mathrm{~g}, 0.005 \mathrm{~mol})$ as long-chain passivator and octadecene (15 mL) as solvent. Typically, PA, 1hexadecylamine and octadecene were transferred into a $100 \mathrm{~mL}$ Teflon-lined stainless-steel autoclave and heated at $200{ }^{\circ} \mathrm{C}$ for $3 \mathrm{~h}$. After cooling to room temperature, the final products were purified by precipitating with acetone three times and further purified by column chromatography. The obtained powder LCQDs were redispersed in toluene to prepare L-CQDs toluene solution $\left(1 \mathrm{mg} \mathrm{mL}^{-1}\right)$ for further use.

\section{Fabrication of CQD-LEDs}

ITO-coated substrate was cleaned ultrasonically in deionized water, acetone in twice and isopropanol for $20 \mathrm{~min}$ each. After cleaning, it was treated by UV-ozone for $10 \mathrm{~min}$. During these treatments the ITO surfaces are exposed to reactive oxygen species. The oxygen-enriched surfaces were achieved, particularly with the formation of peroxides. A downward shift of the Fermi level is obtained by reducing the charge distribution density at or near the surface via oxygen implantation. The shift of the Fermi level contributes to the increase in the ITO work function. The formed surface peroxide species lead to an

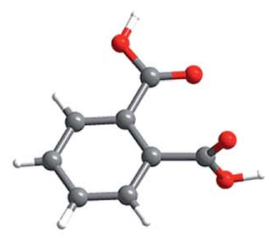

PA

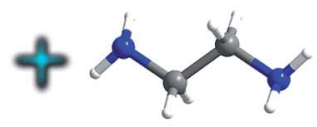

EDA
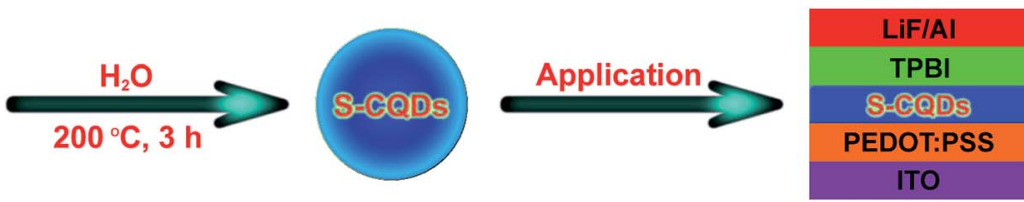

CQD-LEDS

Scheme 1 Schematic illustration of synthesizing S-CQDs for CQD-LEDs. 
increased ionization potential (surface dipole), which also contributes to the increase in the work function. ${ }^{28}$ PEDOT:PSS was then spin-coated on the UV-ozone treated ITO surface to give a $40 \mathrm{~nm}$-thick film at $3000 \mathrm{rpm}$ for $60 \mathrm{~s}$ with $180 \mu \mathrm{L}$ of PEDOT:PSS solution, followed by annealing in an oven at $120^{\circ} \mathrm{C}$ for $20 \mathrm{~min}$ in air. Subsequently, the emissive layer of S-CQDs was spin-coated from the toluene solution on the surface of PEDOT:PSS at $1000 \mathrm{rpm}$ for $30 \mathrm{~s}$ with $360 \mu \mathrm{L}$ of CQD toluene solution and annealed at $120{ }^{\circ} \mathrm{C}$ for $20 \mathrm{~min}$. Then, $40 \mathrm{~nm}$-thick TPBI, $1 \mathrm{~nm}$-thick LiF, and $100 \mathrm{~nm}$-thick aluminum were sequentially deposited in thermal evaporator under high vacuum $\left(5 \times 10^{-4} \mathrm{~Pa}\right)$. For hole- and electron-only devices, the parameters of L-CQDs spin-coating and annealing are the same as those for S-CQDs. For hole-only devices, $40 \mathrm{~nm}$-thick $N, N^{\prime}$-bis-(1-naphthalenyl)- $N, N^{\prime}$-bis-phenyl-(1,1'-biphenyl)-4, $4^{\prime}$ diamine (NPB) was deposited in thermal evaporator under high vacuum $\left(5 \times 10^{-4} \mathrm{~Pa}\right)$.

\section{Characterization}

Ultraviolet-visible (UV-vis) absorption and emission spectra were recorded on a Hitachi U3900 UV-vis spectrophotometer and a Horiba Fluoromax-4 luminescence spectrometer. The fluorescence lifetime of the S-CQDs was measured with Transient Fluorescence \& Phosphorescence (FLS980). The QY of SCQDs was obtained by a comparative method. Quinine sulfate (QY $=54 \%$ ) in $0.10 \mathrm{M} \mathrm{H}_{2} \mathrm{SO}_{4}$ was chosen as the reference to determine the QY of S-CQDs in aqueous solution at different concentration. All the absorbance values of the solutions were measured at $360 \mathrm{~nm}$. Absorbance values were kept under 0.1 at the excitation wavelength for minimizing re-absorption effects. The integrated emission intensity is the area under the emission curve from 380 to $700 \mathrm{~nm}$. The linear fitting lines of the integrated emission intensity against the absorbance were plotted. Finally, QY was calculated as follows (1):

$$
Q=Q_{\mathrm{st}}\left(K / K_{\mathrm{st}}\right)\left(\eta / \eta_{\mathrm{st}}\right)^{2}
$$

where $Q$ is $\mathrm{QY}, K$ is the slope of the linear fitting line, $\eta$ is refractive index of the solvent. For the solutions in the present study, $\eta / \eta_{\text {st }}=1$. The subscript "st" refers to quinine sulfate standard solution. Transmission electron microscopy (TEM) images were taken on a JEOL JEM-2100 microscope. X-ray diffraction (PXRD) patterns were recorded on a Rigaku-D/MAX 2500 diffractometer with graphite monochromatized $\mathrm{Cu} \mathrm{K} \alpha(\lambda$ $=0.15405 \mathrm{~nm}$ ) radiation. Fourier transform infrared (FTIR) spectra were measured with a Bruker Tensor 27 spectrometer. Xray photoelectron spectroscopy (XPS) measurements were performed on a Kratos AXIS ULTRA DLD X-ray photoelectron spectrometer with mono X-ray source Al K $\alpha$ excitation (1486.6 $\mathrm{eV}$ ). Cyclic voltammetry (CV) curve was acquired in a tetrabutylammonium hexafluorophosphate (TBAP, $0.1 \mathrm{~mol} \mathrm{~L}^{-1}$ ) DMF solution using a CHI600E electrochemical workstation operated at a scanning rate of $0.1 \mathrm{~V} \mathrm{~s}^{-1}$, in which a Pt disk was used as the working electrode, a Pt wire and a saturated calomel electrode (SCE) served as the counter and reference electrode, respectively, and ferrocene was used to calibrate the redox potentials. The atomic force microscopy (AFM) images of S-CQDs on the surface of PEDOT:PSS were obtained on SPA-300 HV scanning probe microscope. The thickness was measured with a Bruker DektakXT. The EL spectra were analyzed by a Spectra Scan PR 655 photometer. The CIE coordinates are generated when the EL spectra are tested, and the $X$ and $Y$ values of CIE coordinate represent the relative content of red and green, respectively. Current-voltage-luminance $(I-V-L)$ characteristics were measured by a computer-controlled Keithley 2400 source meter integrated with an ST-900M luminance meter.

\section{Results and discussion}

\section{Morphology and structure of the S-CQDS}

The morphology and microstructure of the S-CQDs were characterized by TEM. As is shown in Fig. 1(a), S-CQDs are quasispherical and well dispersed without obvious aggregation, the majority of the particles fall in the range of 4 to $7 \mathrm{~nm}$ in diameter. According to the size distribution histogram (inset in Fig. 1(a)), the average particle size of these CQDs is calculated to be $5.53 \mathrm{~nm}$. The HRTEM image shows that S-CQDs have wellresolved lattice fringes (inset in Fig. 1(b)). The lattice spacing of $0.22 \mathrm{~nm}$ agrees well with the (100) diffraction facets of graphite. ${ }^{29,30}$ The results illustrate that the S-CQDs are structured like crystalline graphite carbon. As shown in Fig. 1(c), PXRD pattern was acquired to further analyze the crystallinity of S-CQDs. A broad diffraction peak at $2 \theta=21.4^{\circ}$ is consistent with the (002) facet of graphite structure. The interlayer spacing of (002) diffraction facet is enlarged by the presence of surface functional groups, which is calculated to be $0.42 \mathrm{~nm}$, larger than that of graphite $(0.33 \mathrm{~nm}) .{ }^{29}$

The polymer-like intermediates are obtained by dehydration polymerization of EDA and PA, and finally carbonized through intramolecular dehydration of the intermediates to form SCQDs with EDA as short chain ligand. To analyze the surface chemical structure of S-CQDs, FTIR spectra of PA, EDA and SCQDs were obtained (Fig. 1(d)). For PA, the stretching of $\mathrm{O}-\mathrm{H}$ at 2649 and $2522 \mathrm{~cm}^{-1}$ originates from $-\mathrm{COOH}$. The stretching of $\mathrm{C}=\mathrm{O}$ and $\mathrm{C}-\mathrm{OH}$ at 1696 and $1279 \mathrm{~cm}^{-1}$ also stems from $-\mathrm{COOH}$ groups. For EDA, the bands at 2855 and $2927 \mathrm{~cm}^{-1}$ represent the stretching of $-\mathrm{CH}$ and $-\mathrm{CH}_{2}$, respectively. The bands at 1160 and $3375 \mathrm{~cm}^{-1}$ originate from the stretching of $\mathrm{C}-\mathrm{N}$ and $-\mathrm{NH}_{2}$, respectively. In the FTIR spectra of PA and SCQDs, the broad bands at 3007 and $3027 \mathrm{~cm}^{-1}$ are attributed to the stretching vibration of $=\mathrm{C}-\mathrm{H}$ in aromatic hydrocarbon, the bands at 1406 and $1378 \mathrm{~cm}^{-1}$ represent the stretching of CH. For S-CQDs, the characteristic absorption bands at 3267 , 1649 and $1550 \mathrm{~cm}^{-1}$ represent the stretching of $\mathrm{N}-\mathrm{H}$ and $\mathrm{C}=\mathrm{O}$ in $-\mathrm{CONH}$ and $\mathrm{C}=\mathrm{C}$ in aromatic ring, respectively. The band at $3393 \mathrm{~cm}^{-1}$ originates from the $-\mathrm{NH}_{2}$ groups of S-CQD surfaces. For S-CQDs, the characteristic absorption bands at 3267, 1649 and $1550 \mathrm{~cm}^{-1}$ represent the stretching of $\mathrm{N}-\mathrm{H}$ and $\mathrm{C}=\mathrm{O}$ in amide bond and $\mathrm{C}=\mathrm{C}$ in aromatic ring, respectively. The band at $3393 \mathrm{~cm}^{-1}$ originates from the surface $-\mathrm{NH}_{2}$ groups of $\mathrm{S}$ CQDs. The results indicate that $-\mathrm{CONH}$ is introduced to $\mathrm{S}$ CQDs by dehydration between $-\mathrm{NH}_{2}$ and $-\mathrm{COOH}$ groups, finally short-chain ligand is grafted on the surface of S-CQDs by dehydration of one of $-\mathrm{NH}_{2}$ groups in EDA. ${ }^{31}$ 
(a)
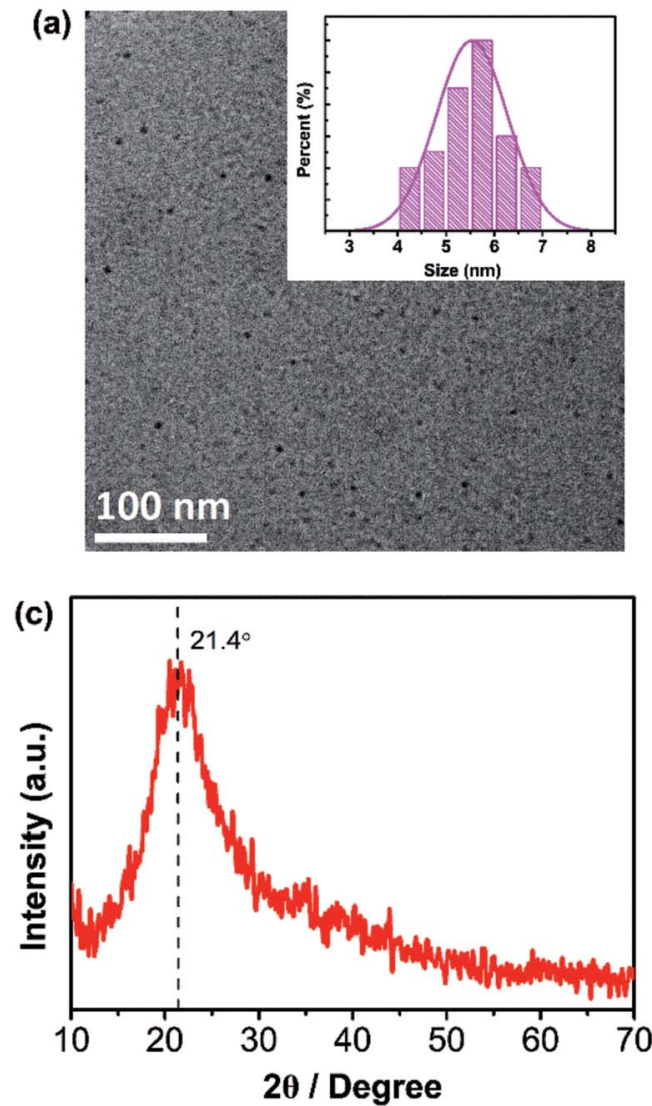

(b)

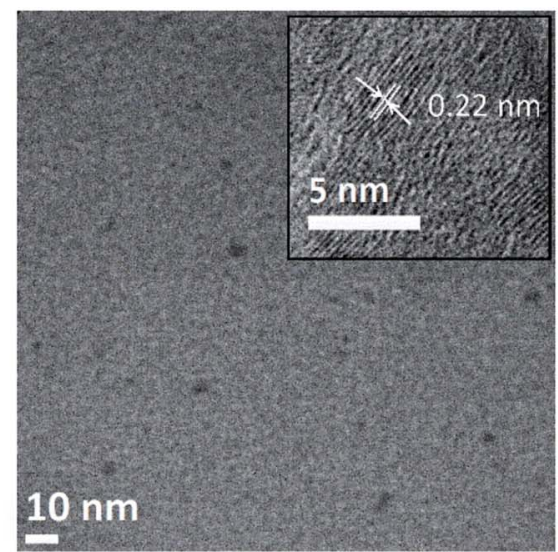

(d)

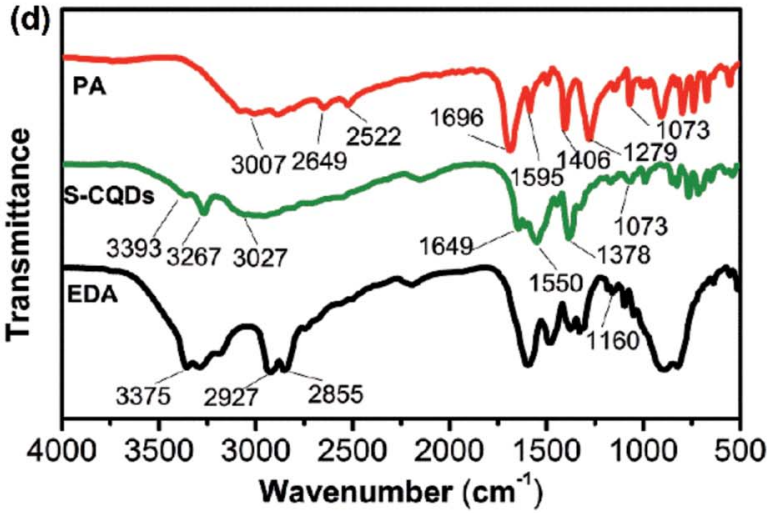

Fig. 1 (a) TEM image of S-CQDs (inset is their size distribution) and (b) the HRTEM image of S-CQDs. (c) PXRD pattern of S-CQDs and (d) FTIR spectra of PA, S-CQDs and EDA.

XPS spectra were also measured to explore the surface structure of S-CQDs (Fig. 2). The full scan XPS spectra of the SCQDs show three peaks at 285 (C1s), 400 (N1s) and $531 \mathrm{eV}$ (O1s) (Fig. 2(a)). The C1s spectra (Fig. 2(b)) have four peaks at 284.7 $(\mathrm{C}=\mathrm{C} / \mathrm{C}-\mathrm{C}), 285.5(\mathrm{C}-\mathrm{N}), 286.4(\mathrm{C}-\mathrm{O})$ and $288.0(\mathrm{C}=\mathrm{O}) \mathrm{eV}$. The N1s spectra (Fig. 2(c)) exhibit three peaks at 399.7, 400.6 and $401.4 \mathrm{eV}$, which correspond to aromatic $\mathrm{C}-\mathrm{N}, \mathrm{N}-\mathrm{C}=\mathrm{O}$ and $\mathrm{N}-\mathrm{H}$, respectively. The aromatic $\mathrm{C}-\mathrm{N}$ and $\mathrm{N}-\mathrm{C}=\mathrm{O}$ signal peaks also show that EDA is involved in the formation of S-CQDs by dehydration between $-\mathrm{NH}_{2}$ and $-\mathrm{COOH}$ groups. ${ }^{32}$ The O1s (Fig. 2(d)) spectra present two peaks at 530.6 (C-O) and 531.3 $(\mathrm{C}=\mathrm{O}) \mathrm{eV}$, which reveal the presence of carboxylic group. The atom ratio of $\mathrm{C}: \mathrm{O}: \mathrm{N}$ is also calculated as $58.3: 30.2: 11.5$ by XPS spectra. ${ }^{33}$

\section{Optical properties of S-CQDs}

To explore the optical properties of S-CQDs, UV-vis absorption and PL spectra were measured. As shown in Fig. 3(a), UV-vis absorption spectra exhibit two obvious absorption peaks at 274 and $308 \mathrm{~nm}$. The strong absorption peak at $274 \mathrm{~nm}$ is attributed to $\pi-\pi^{*}$ transition of aromatic $\mathrm{C}=\mathrm{C}$ bond $^{34-37}$ and the relatively weak absorption peak at $308 \mathrm{~nm}$ corresponds to $\mathrm{n}-$ $\pi^{*}$ transition of $\mathrm{C}=\mathrm{O}$ bond. ${ }^{33,38}$ The two absorption peaks indicate the existence of conjugated structures and oxygencontaining functional groups in S-CQDs. The PL spectra of SCQD aqueous solution in Fig. 3(a) show a strong peak at
$439 \mathrm{~nm}$ upon excitation at $360 \mathrm{~nm}$. The S-CQD aqueous solution emits colourless under daylight (inset left in Fig. 3(a)) but blue light under $365 \mathrm{~nm}$ UV lamp (inset right in Fig. 3(a)).

The S-CQDs exhibit excitation-dependent PL emission (Fig. 3(b)), which is possibly resulted from the nonuniform size and diversified surface sates of S-CQDs. ${ }^{39}$ The PL intensity of SCQD aqueous solutions decreases as the excitation wavelength
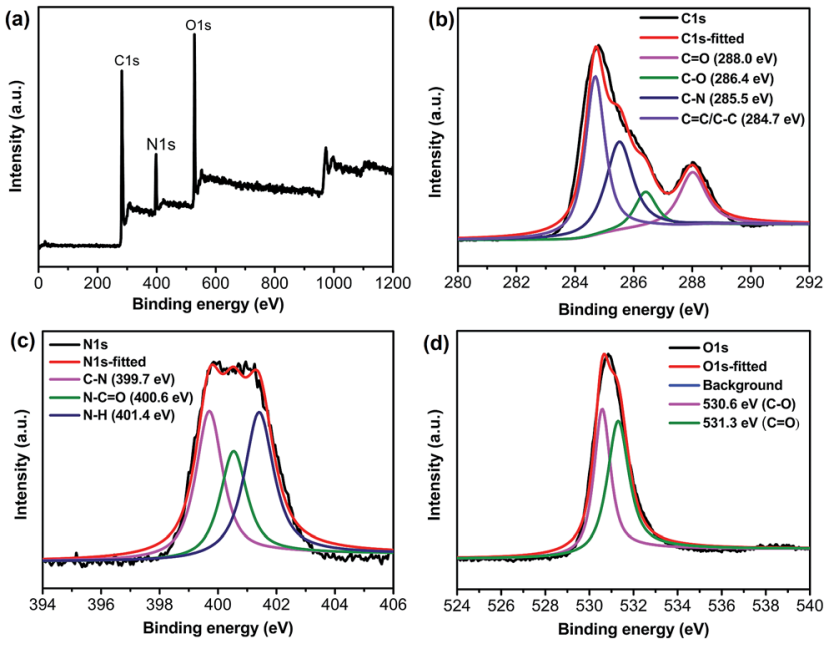

Fig. 2 (a) XPS spectra and corresponding expansion for (b) C1s, (c) N1s and (d) O1s peaks of S-CQDs. 

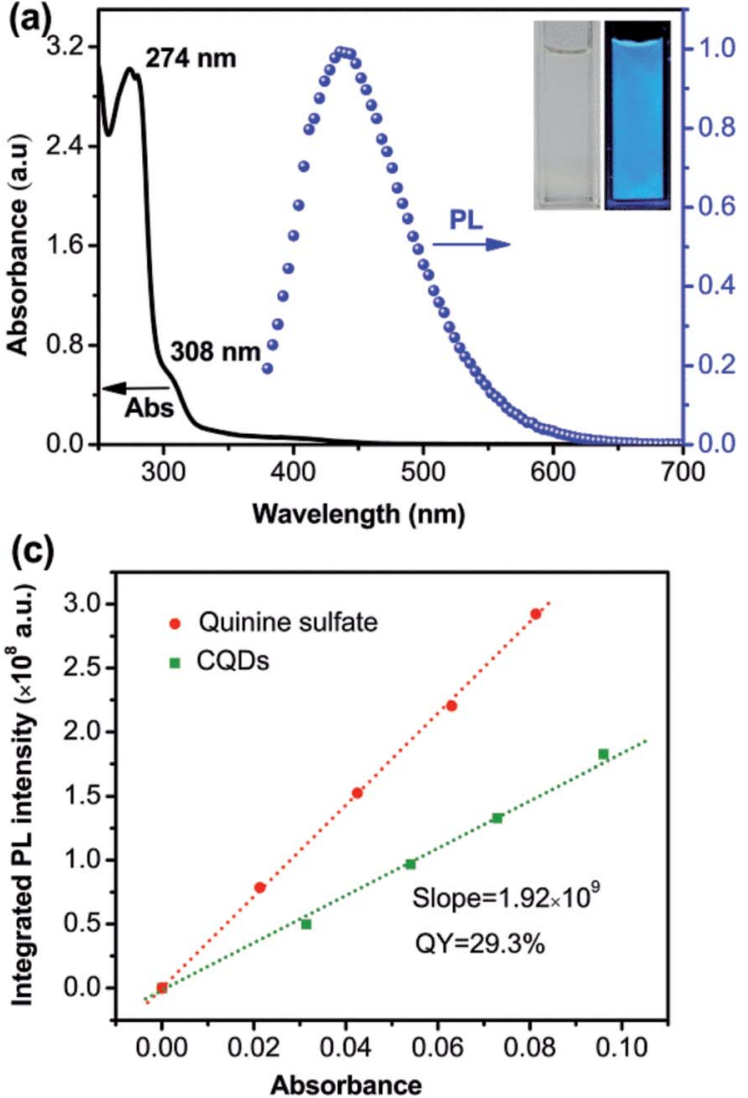

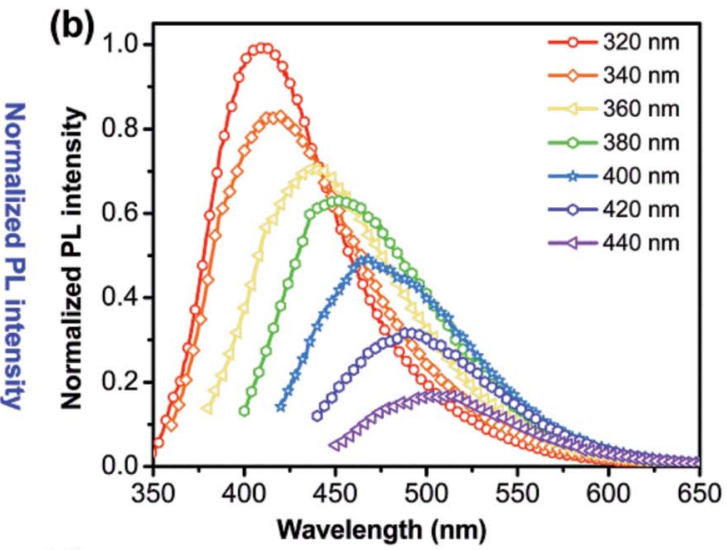

(d)

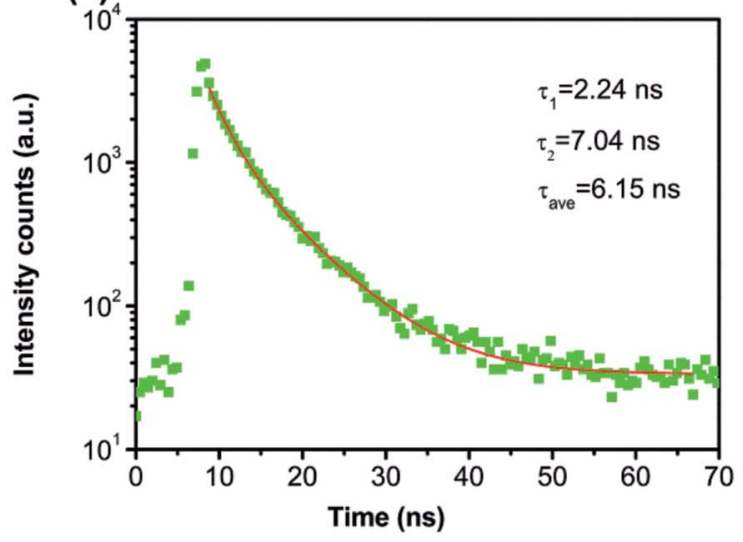

Fig. 3 (a) UV-vis absorption spectra of CQD aqueous solution and PL spectra of CQD aqueous solution at excitation wavelength of $365 \mathrm{~nm}$. Inset: the photograph of S-CQDs aqueous solution under daylight (left) and UV light (right). (b) PL spectra of S-CQDs under different excitation wavelengths. (c) QY diagram and (d) fluorescence-decay and fitted curves of S-CQDs.

increases from 320 to $440 \mathrm{~nm}$. The emission centers denote the surface states of CQDs. ${ }^{40}$ CQDs have many emission centers because of different surface chemical groups on CQDs. The distribution of emission centers on CQDs can be reflected by their PL spectra. At certain excitation wavelength, the corresponding emission center could be excited to exhibit fluorescence, resulting in the excitation-dependent behaviors of PL spectra. ${ }^{41,42}$ The dominant emission center, corresponding to the emission peak, indicates the emission center as a leading role under certain excitation wavelength. Only a small portion of the deeper surface states can be excited by long wavelength because the excitation energy is smaller than the band gap of the dominant emission center. ${ }^{43}$ Eventually, the fluorescence decreases greatly. In addition, the emission wavelength exhibits a red-shift with the increase of excitation wavelength. Different functional groups may act as different emission centers to emit light. The dominant emission center may transfer to the emission center of smaller band gap with the increase of excitation wavelength..$^{43}$ The energy of longer wavelength is mainly used for exciting the longer wavelength emission with smaller band gap, being not enough to excite shorter wavelength emission with bigger band gap. ${ }^{25}$ Consequently, the emission peak is at the long wavelength when the excitation energy is low; while it is at the short wavelength when the excitation energy is high.
The QY was tested with quinine sulfate as a standard (QY = $54 \%$ ). As shown in Fig. 3(c), the QY of the S-CQDs is calculated as $29.3 \%$. Over many experiments, the $\mathrm{COOH}$ and $\mathrm{NH}_{2}$ groups in reactants were found to be very important for the QY of CQDs. The QY depends on the competition between the emissive sites and non-radiative traps..$^{22}$ The $\mathrm{COOH}$ groups serve as non-radiative recombination centers of electron-hole pairs, ${ }^{40}$ the nitrogen-doping introduces a kind of surface state (such as amino or amido bond). Electrons trapped by the nitrogen doped surface states are able to depress the effect of non-radiative recombination and accelerate that of radiative recombination. ${ }^{39,44}$ The active $\mathrm{COOH}$ groups can combine more $\mathrm{NH}_{2}$ groups to increase the QY of S-CQDs. ${ }^{45}$ Ultimately, S-CQDs with high QY are likely to favor the optical performance of CQDLEDs. ${ }^{24}$ Furthermore, the QY is expected to be further improved by optimizing synthesis parameters ${ }^{22,46}$ and co-doping with nitrogen and sulfur..$^{39,47}$

The fluorescence decay lifetime of S-CQDs was estimated under the excitation of $375 \mathrm{~nm}$ and the emission of $455 \mathrm{~nm}$ by using multidimensional time-correlated single photon counting (TCSPC) method. As depicted in Fig. 3(d), the decay trace of S-CQDs was fitted using bi-exponential function in eqn (2):

$$
R(t)=B_{1} \exp \left(-t / \tau_{1}\right)+B_{2} \exp \left(-t / \tau_{2}\right)
$$


where $B_{1}$ and $B_{2}$ are the pre-exponential factors for the time resolved decay lifetime of $\tau_{1}$ and $\tau_{2}$, respectively. The average lifetime is calculated by eqn (3):

$$
\tau=\frac{B_{1} \tau_{1}^{2}+B_{1} \tau_{2}^{2}}{B_{1} \tau_{1}+B_{2} \tau_{2}}
$$

The test results show that decay curve has two lifetime components of $2.24\left(\tau_{1}\right)$ and $7.04 \mathrm{~ns}\left(\tau_{2}\right)$, and the corresponding pre-exponential factors are $0.05\left(B_{1}\right)$ and $0.07\left(B_{2}\right)$, respectively. The average lifetime of S-CQDs is calculated to be $6.15 \mathrm{~ns}$. The slow decay is due to the effective relaxation of carriers from carbogenic core onto the surface states and the fast decay is attributed to the recombination of carriers on the surface states. ${ }^{43}$

\section{HOMO and LUMO energy levels of S-CQDS}

Apart from the optical property, S-CQDs can also serve as a good electron donor and acceptor. ${ }^{\mathbf{2 4 , 2 5}}$ To acquire the highest occupied molecular orbital (HOMO) and the lowest unoccupied molecular orbital (LUMO) energy levels, cyclic voltammetry measurements were implemented. The CV curves of $0.1 \mathrm{~mol} \mathrm{~L}^{-1}$ TBAP-DMF solution and $0.1 \mathrm{~mol} \mathrm{~L}^{-1}$ TBAP-DMF with S-CQDs solution are shown in Fig. 4(a), in which the S-CQDs exhibit apparently a reversible reduction process. By extrapolating the CV curve of TBAP-DMF solution with S-CQDs, the onset reduction potential ( $E_{\text {red }}^{\text {onset }}$ ) was determined to be $-0.65 \mathrm{~V} v s$. SCE. By extrapolating the $(a h \nu)^{2}-h \nu$ (where $a$ : absorbance, $h$ : Plank's constant, and $\nu$ : frequency) curve (Fig. $4(\mathrm{~b})$ ), the band gap $\left(E_{\mathrm{g}}\right)$ of the S-CQDs was estimated to be $4.26 \mathrm{eV}$ (Fig. 4(b)). ${ }^{48}$ The LUMO and HOMO energy levels ( $E_{\mathrm{LUMO}}$ and $E_{\mathrm{HOMO}}$ ) were then calculated to be -4.09 and $-8.35 \mathrm{eV}$, respectively, by eqn (4): ${ }^{15}$

$$
E_{\mathrm{LUMO}}=-e\left(E_{\mathrm{red}}^{\mathrm{onset}}+4.74\right), E_{\mathrm{HOMO}}=E_{\mathrm{LUMO}}-E_{\mathrm{g}}
$$

\section{CQD-LEDs demonstration}

To investigate the film-forming ability of S-CQDs, the AFM images of $40 \mathrm{~nm}$-thick PEDOT:PSS and S-CQDs atop of $40 \mathrm{~nm}$ -
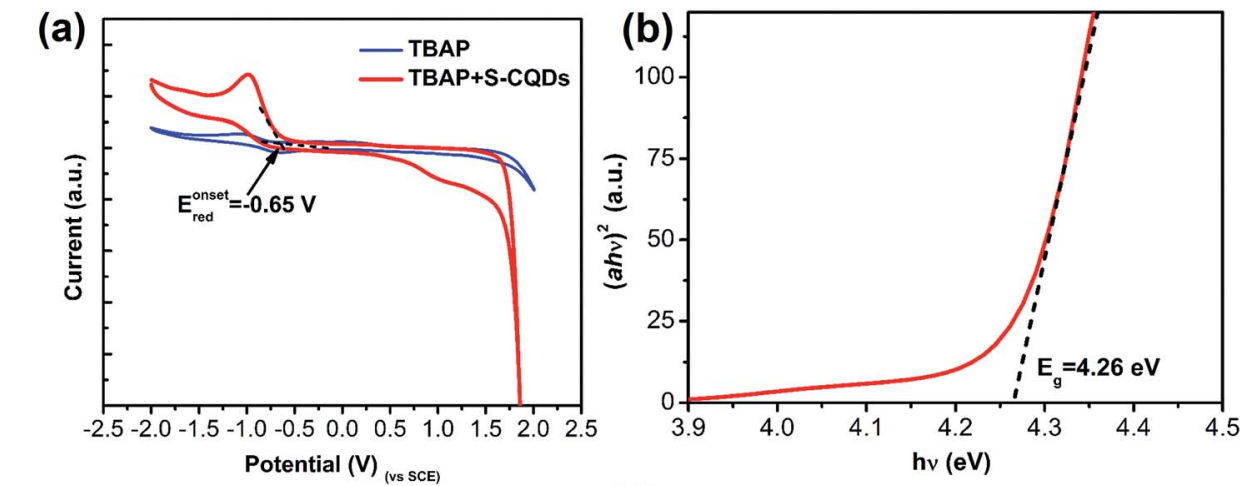

(c)
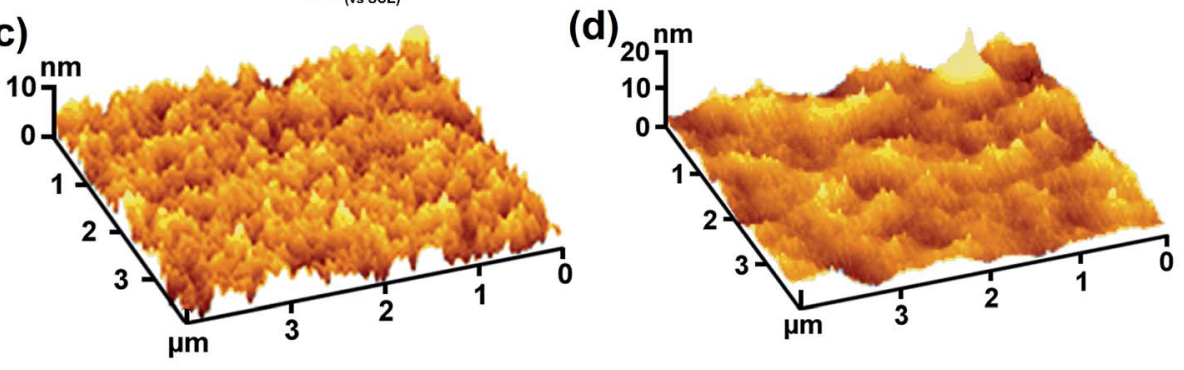

(e)

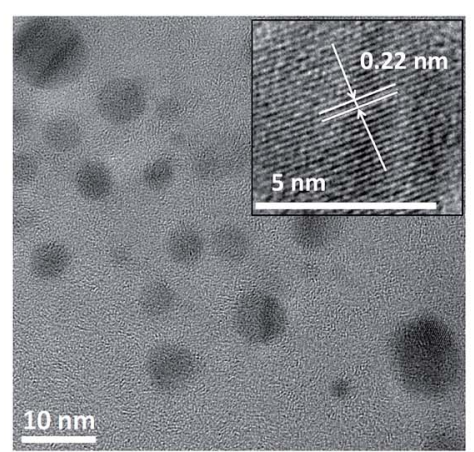

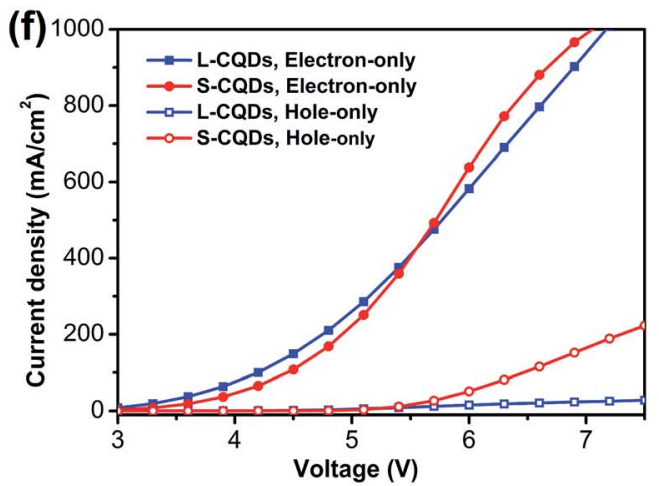

Fig. 4 (a) Cyclic voltammogram of the $0.1 \mathrm{~mol} \mathrm{~L}^{-1}$ TBAP-DMF solution and the S-CQDs in $0.1 \mathrm{~mol} \mathrm{~L}^{-1}$ TBAP-DMF solution at a scan rate of $0.1 \mathrm{~V}$ $\mathrm{s}^{-1}$. (b) The dependence of $(a h \nu)^{2}$ on $h \nu$ for the S-CQDs, from which a band gap of $4.26 \mathrm{eV}$ is deduced. AFM images of (c) $40 \mathrm{~nm}$-thick PEDOT:PSS surface and (d) S-CQDs surface atop of $40 \mathrm{~nm}$-thick PEDOT:PSS. (e) TEM image (inset is HRTEM image) of L-CQDs. (f) Current-density-voltage characteristics of electron- and hole-only devices based on L-CQDs and S-CQDs. 
thick PEDOT:PSS are shown in Fig. 4(c) and (d), respectively. Nearly uniform and flat films are presented without massive aggregation. The average surface roughness $\left(R_{\mathrm{a}}\right)$ of PEDOT:PSS films (Fig. 4(c)) and S-CQDs films (Fig. 4(d)) was calculated to be 1.313 and $2.235 \mathrm{~nm}$, respectively. The root mean square roughness (RMS) of PEDOT:PSS films (Fig. 4(c)) and S-CQD films (Fig. 4(d)) was calculated to be 1.647 and $2.997 \mathrm{~nm}$, respectively. The results prove that S-CQDs in toluene solution are beneficial to the dispersion of S-CQD particles to form high-quality film. The aggregate morphology between S-CQD particles has an effect on the transmission and radiation of carriers. This can be explained by the luminous quenching and the poor charge transportation arising from intensive distribution and sparse distribution of S-CQD particles, respectively. Therefore, an appropriate dispersion density of S-CQD particles is required.

In order to compare the carrier injection ability of S-CQDs with that of L-CQDs, L-CQDs were prepared by one-step solvothermal treatment using PA as carbon source, 1-hexadecylamine as long-chain passivator and octadecene as solvent. Fig. 4(e) shows that L-CQDs also have similar graphitic structure with S-CQDs. Single carrier devices with only electrons or holes moving through CQDs are fabricated and characterized to compare the carrier injection ability of S-CQDs and L-CQDs. The electron-only devices have a multilayer structure of ITO/CQDs/ TPBI $(40 \mathrm{~nm}) / \mathrm{LiF}(1 \mathrm{~nm}) / \mathrm{Al}(100 \mathrm{~nm})$. The hole-only devices have a structure of ITO/PEDOT:PSS (40 nm)/CQDs/NPB (40 nm)/ $\mathrm{Al}(100 \mathrm{~nm})$, where PEDOT:PSS and NPB is hole injection layer.
In the hole-only devices, the current represents the hole transport behavior for the device. ${ }^{49}$ From the current density-voltage $(J-V)$ characteristics of the electron-only and hole-only devices shown in Fig. 4(f), we see that both the electron and hole currents of S-CQDs are higher than those of L-CQDs at voltage range of 5.5-7 V, especially for the hole current. The results indicate that S-CQDs can improve the injection ability of electrons and holes. The surface of L-CQDs may form an organic insulating layer larger than $0.5 \mathrm{~nm}$ in thickness, which would forms a minimal tunneling barrier to hinder the injection of electrons and holes. ${ }^{6}$ Compared with the L-CQDs, the S-CQDs have no insulating layer of larger than $0.5 \mathrm{~nm}$ thickness on their surfaces. The absence of the tunneling barrier reduces the obstacle of carrier injection, thus improving the carrier injection ability.

To further explore the electroluminescent properties of $\mathrm{S}$ CQDs, S-CQDs were used as the emitting layer to fabricate CQD-LEDs consisting of a patterned ITO anode, a $40 \mathrm{~nm}$-thick PEDOT:PSS hole injection layer, a $40 \mathrm{~nm}$-thick TPBI electron transport layer, a $1 \mathrm{~nm}$-thick LiF and $100 \mathrm{~nm}$-thick $\mathrm{Al}$ cathode. Electrons are injected from the cathode into the ETL, holes are injected from ITO anode into the HIL and then the carriers travel towards the layer of S-CQDs. Finally, the carriers recombine in S-CQDs to emit photons. ${ }^{6}$ The energy level diagram of SCQDs is shown in Fig. 5(a).

The electroluminescence spectra of the device driven at forward voltage (6-9 V) are shown in Fig. 5(b). The EL peak is
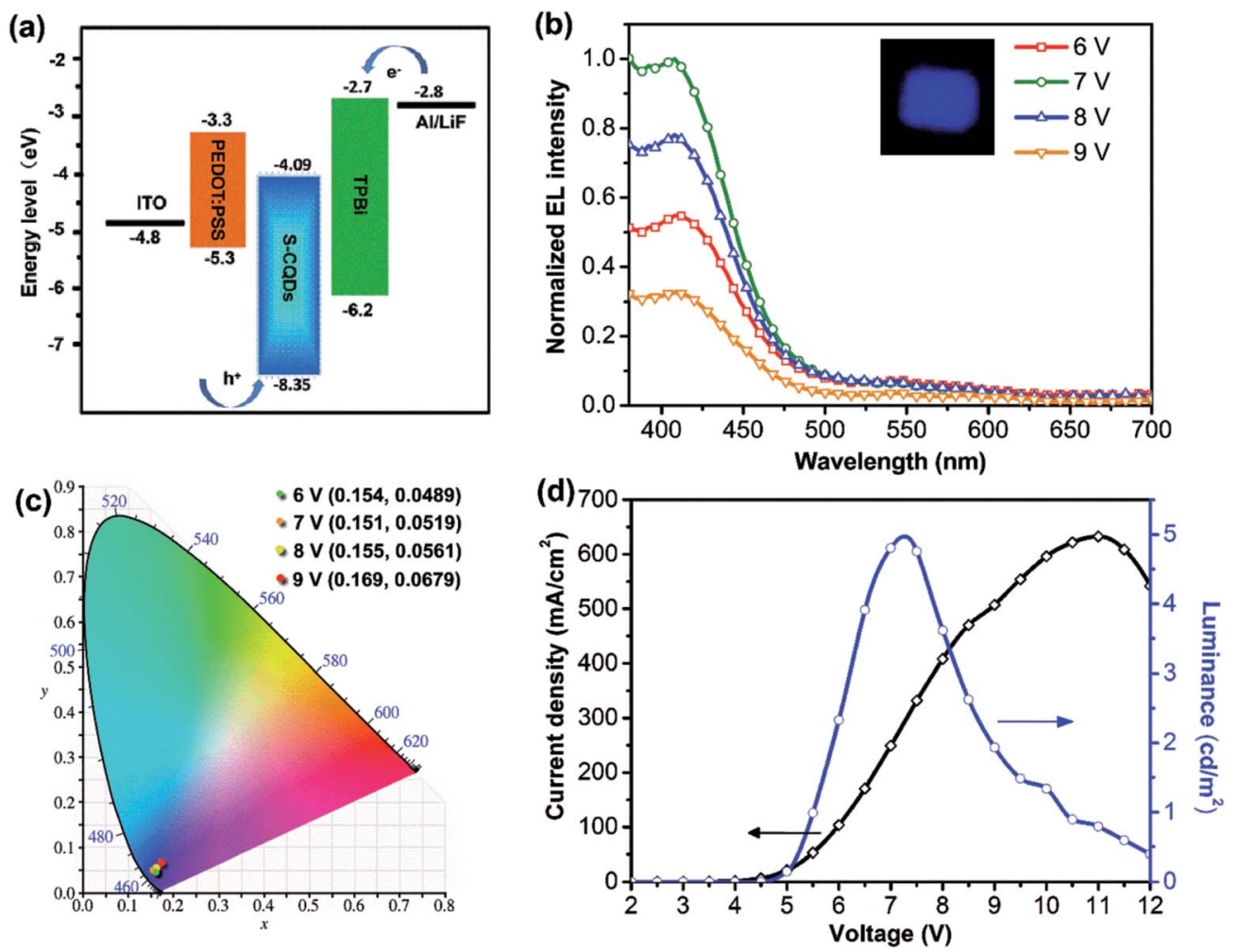

Fig. 5 (a) The schematic energy level diagram of the CQD-LEDs. (b) Emission spectra of CQD-LEDs under different working voltage (inset is the photograph of CQD-LEDs under the working voltage of $7 \mathrm{~V}$ ). (c) The CIE 1931 chromaticity coordinates of CQD-LEDs under different working voltage. (d) The current density curve and brightness-voltage characteristics of CQD-LEDs. 
positioned without apparent variation and remains at around $410 \mathrm{~nm}$. The CQD-LEDs emit bright blue light at 6-9 V. The EL peak intensity of these devices first increases, reaches maximum at voltage of $7 \mathrm{~V}$ over $6-9 \mathrm{~V}$, and then decreases. More charges are injected into the S-CQDs with the increase of current density. The generation probability of photon is increased by more excitons generated from electrons and holes. Consequently, the EL intensity increases. Charge imbalance caused by excessive current may result in the decrease of EL intensity. In addition, as the injection current density is low at the driving voltage of 6-9 V, the carriers preferentially relax via the energy level associated with blue light emitting. ${ }^{25}$

Therefore, EL peak of the CQD-LEDs remains at the same blue light wavelength when the driving voltage is in the range of 6-9 V. As shown in Fig. 5(c), the corresponding CIE coordinates exhibit no obvious variation with the voltage changing from 6 to $9 \mathrm{~V}$, which shows a relatively good optical stability at the applied voltages.

The current density dramatically increases with the change of driven voltage from $5.5-11 \mathrm{~V}$ and reaches the maximum value $\left(630 \mathrm{~mA} \mathrm{~cm}^{-2}\right)$ at $11 \mathrm{~V}$ (Fig. $\left.5(\mathrm{~d})\right)$. The turn-on voltage is about $5.5 \mathrm{~V}$. The brightness increases greatly with the change of driven voltage from 5.5-7.26 V and the maximum luminance reaches $4.97 \mathrm{~cd} \mathrm{~m}^{-2}$. The performance of the CQD-LEDs such as the maximum luminance is still lower than that of QD-LEDs fabricated by semiconductor QDs. ${ }^{49}$ The maximum luminance of the device is also lower than that of blue emitting CQD-LEDs (maximum brightness is $24 \mathrm{~cd} \mathrm{~m}^{-2}$ ) based on long-chain passivated CQDs synthesized by thermally carbonizing citric acid in hot octadecene with 1-hexadecylamine as the passivation agent. ${ }^{25}$ The QY of long-chain passivated CQDs was determined to be $\approx 40 \%$ by using a spectrometer with an integrating sphere. There are two reasons that can be used to explain the phenomenon. First, the carrier transfer ability of SCQDs is improved by the short-chain modification of ethylenediamine and the graphitic structure. But the lower QY of SCQDs compared with that of long-chain passivated CQDs ${ }^{25}$ may weaken the optical performance of CQD-LEDs. ${ }^{24}$ Second, highquality film can be formed by the S-CQDs from toluene solution. However, electrons are injected smoothly from the cathode and holes encounter barriers during the injection process because of the large energy barrier $(3.05 \mathrm{eV})$ between the luminescent layer and HIL, which results in an unbalanced charge injection. ${ }^{7}$ Charge balance is an important factor for realizing high-performance CQD-LEDs. ${ }^{7}$ Hence, to improve the performance of CQD-LEDs, the QY of S-CQDs should be improved by further optimizing synthesis parameters and codoping with nitrogen and sulfur, suitable device structure also should be chosen to reduce the potential barrier.

\section{Conclusions}

In conclusion, S-CQDs with graphitic structure were synthesized through a facile hydrothermal method using PA and EDA as the precursor and modifier, respectively. The obtained SCQDs have an average diameter of $5.53 \mathrm{~nm}$, exhibit excitationdependent PL emission and possess QY of $29.3 \%$ in aqueous solutions. The structural analyses of the S-CQDs by FTIR and XPS demonstrate both PA and EDA have engaged in the establishment of S-CQDs. The S-CQDs have electron-accepting ability, as proved by electrochemical analysis. The CQD films were nearly uniform and flat when CQD-LEDs were fabricated by using S-CQDs as emitting layer. The device exhibits good optical stability under the voltage of 6-9 V, a turn-on voltage at about $5.5 \mathrm{~V}$ and a maximum luminance of $4.97 \mathrm{~cd} \mathrm{~m}^{-2}$. The reasons for the low brightness are speculated to be the relatively low QY of S-CQDs and the large energy barrier $(3.05 \mathrm{eV})$ between the emitting layer and the HIL. The performance of the CQDLEDs is anticipated to be further improved by choosing the SCQDs with high QY and suitable device structure with low energy barrier.

\section{Acknowledgements}

This work was supported by the Shanxi Provincial Key Innovative Research Team in Science and Technology (201605D131045-10) and Shanxi Provincial Key Research and Development Program (201603D111010), National Natural Science Foundation of China (U1610255, U1607120).

\section{Notes and references}

1 Q. Sun, Y. A. Wang, L. S. Li, D. Wang, T. Zhu, J. Xu, C. Yang and Y. Li, Nat. Photonics, 2007, 1, 717-722.

2 J. S. Steckel, P. Snee, S. Coe-Sullivan, J. P. Zimmer, J. E. Halpert, P. Anikeeva, L.-A. Kim, V. Bulovic and M. G. Bawendi, Angew. Chem., Int. Ed., 2006, 45, 5796-5799.

3 X. Michalet, F. F. Pinaud, L. A. Bentolila, J. M. Tsay, S. Doose, J. J. Li, G. Sundaresan, A. M. Wu, S. S. Gambhir and S. Weiss, Science, 2005, 307, 538-544.

4 M. A. Schreuder, K. Xiao, I. N. Ivanov, S. M. Weiss and S. J. Rosenthal, Nano Lett., 2010, 10, 573-576.

5 A. Rizzo, M. Mazzeo, M. Biasiucci, R. Cingolani and G. Gigli, Small, 2008, 4, 2143-2147.

6 P. O. Anikeeva, J. E. Halpert, M. G. Bawendi and V. Bulovic, Nano Lett., 2009, 9, 2532-2536.

7 T. Ding, X. Yang, L. Ke, Y. Liu, W.-Y. Tan, N. Wang, X.-H. Zhu and X. W. Sun, Org. Electron., 2016, 32, 89-93.

8 S. Coe, W.-K. Woo, M. Bawendi and V. Bulović, Nature, 2002, 420, 800-803.

9 Y. Shirasaki, G. J. Supran, M. G. Bawendi and V. Bulović, Nat. Photonics, 2013, 7, 13-23.

10 Q. Sun, Y. A. Wang, L. S. Li, D. Wang, T. Zhu, J. Xu, C. Yang and Y. Li, Nat. Photonics, 2007, 1, 717-722.

11 H. V. Demir, S. Nizamoglu, T. Erdem, E. Mutlugun, N. Gaponik and A. Eychmüller, Nano Today, 2011, 6, 632647.

12 W.-S. Song, J.-H. Kim, J.-H. Lee, H.-S. Lee, Y. R. Do and H. Yang, J. Mater. Chem., 2012, 22, 21901-21908.

13 W.-S. Song and H. Yang, Chem. Mater., 2012, 24, 1961-1967.

14 H.-M. Kim, J.-H. Youn, G.-J. Seo and J. Jang, J. Mater. Chem. C, 2013, 1, 1567-1573.

15 F. Wang, Y.-H. Chen, C.-Y. Liu and D.-G. Ma, Chem. Commun., 2011, 47, 3502-3504. 
16 W. Chung, H. Jung, C. H. Lee, S. H. Park, J. Kim and S. H. Kim, J. Electrochem. Soc., 2011, 158, H1218-H1220.

17 W. Chung, H. Jung, C. H. Lee and S. H. Kim, Opt. Express, 2012, 20, 25071-25076.

18 Y.-P. Sun, B. Zhou, Y. Lin, W. Wang, K. S. Fernando, P. Pathak, M. J. Meziani, B. A. Harruff, X. Wang and H. Wang, J. Am. Chem. Soc., 2006, 128, 7756-7757.

19 S. Y. Lim, W. Shen and Z. Gao, Chem. Soc. Rev., 2015, 44, 362381.

20 Y.-H. Chan, J. Chen, Q. Liu, S. E. Wark, D. H. Son and J. D. Batteas, Anal. Chem., 2010, 82, 3671-3678.

21 X. Feng, F. Zhang, Y. Wang, Y. Zhang, Y. Yang and X. Liu, Appl. Phys. Lett., 2015, 107, 213102.

22 F. Zhang, X. Feng, Y. Zhang, L. Yan, Y. Yang and X. Liu, Nanoscale, 2016, 8, 8618-8632.

23 L. M. Veca, A. Diac, I. Mihalache, P. Wang, G. E. LeCroy, E. M. Pavelescu, R. Gavrila, E. Vasile, A. Terec and Y.-P. Sun, Chem. Phys. Lett., 2014, 613, 40-44.

24 S. Do, W. Kwon, Y. H. Kim, S. R. Kang, T. Lee, T. W. Lee and S. W. Rhee, Adv. Opt. Mater., 2016, 4, 276-284.

25 X. Zhang, Y. Zhang, Y. Wang, S. Kalytchuk, S. V. Kershaw, Y. Wang, P. Wang, T. Zhang, Y. Zhao and H. Zhang, ACS Nano, 2013, 7, 11234-11241.

26 L. Bakueva, S. Musikhin, M. Hines, T.-W. Chang, M. Tzolov, G. Scholes and E. Sargent, Appl. Phys. Lett., 2003, 82, 28952897.

27 S. Chaudhary, M. Ozkan and W. C. Chan, Appl. Phys. Lett., 2004, 84, 2925-2927.

28 S. Harvey, T. Mason, Y. Gassenbauer, R. Schafranek and A. Klein, J. Phys. D: Appl. Phys., 2006, 39, 3959.

29 W. F. Zhang, L. M. Jin, S. F. Yu, H. Zhu, S. S. Pan, Y. H. Zhao and H. Y. Yang, J. Mater. Chem. C, 2014, 2, 1525.

30 Y. Wang, S. Kalytchuk, L. Wang, O. Zhovtiuk, K. Cepe, R. Zboril and A. L. Rogach, Chem. Commun., 2015, 51, 2950-2953.

31 W. Lu, X. Gong, Z. Yang, Y. Zhang, Q. Hu, S. Shuang, C. Dong and M. M. F. Choi, RSC Adv., 2015, 5, 16972-16979.
32 S. Hu, A. Trinchi, P. Atkin and I. Cole, Angew. Chem., Int. Ed., 2015, 54, 2970-2974.

33 Y. Zhang, Y. H. He, P. P. Cui, X. T. Feng, L. Chen, Y. Z. Yang and X. G. Liu, RSC Adv., 2015, 5, 40393-40401.

34 S. Qu, X. Wang, Q. Lu, X. Liu and L. Wang, Angew. Chem., Int. Ed., 2012, 51, 12215-12218.

35 H. Li, X. He, Y. Liu, H. Huang, S. Lian, S.-T. Lee and Z. Kang, Carbon, 2011, 49, 605-609.

36 J. Shang, L. Ma, J. Li, W. Ai, T. Yu and G. G. Gurzadyan, Sci. Rep., 2012, 2, 792.

37 L. Tang, R. Ji, X. Cao, J. Lin, H. Jiang, X. Li, K. S. Teng, C. M. Luk, S. Zeng and J. Hao, ACS Nano, 2012, 6, 5102-5110.

38 Y. L. Wang, Y. Q. Zhao, Y. Zhang, F. Zhang, X. T. Feng, L. Chen, Y. Z. Yang and X. G. Liu, RSC Adv., 2016, 6, 38761-38768.

39 Y. Dong, H. Pang, H. B. Yang, C. Guo, J. Shao, Y. Chi, C. M. Li and T. Yu, Angew. Chem., Int. Ed., 2013, 52, 7800-7804.

40 S. Zhu, Y. Song, X. Zhao, J. Shao, J. Zhang and B. Yang, Nano Res., 2015, 8, 355-381.

41 L. Bao, Z. L. Zhang, Z. Q. Tian, L. Zhang, C. Liu, Y. Lin, B. Qi and D. W. Pang, Adv. Mater., 2011, 23, 5801-5806.

42 X. Li, Y. Liu, X. Song, H. Wang, H. Gu and H. Zeng, Angew. Chem., Int. Ed., 2015, 54, 1759-1764.

43 V. Nguyen, J. Si, L. Yan and X. Hou, Carbon, 2015, 95, 659663.

44 D. Qu, M. Zheng, L. Zhang, H. Zhao, Z. Xie, X. Jing, R. E. Haddad, H. Fan and Z. Sun, Sci. Rep., 2014, 4, 5294.

45 S. Zhu, Q. Meng, L. Wang, J. Zhang, Y. Song, H. Jin, K. Zhang, H. Sun, H. Wang and B. Yang, Angew. Chem., Int. Ed., 2013, 52, 3953-3957.

46 Y. Zhang, Y. Wang, X. Feng, F. Zhang, Y. Yang and X. Liu, Appl. Surf. Sci., 2016, 387, 1236-1246.

47 M. Xue, L. Zhang, M. Zou, C. Lan, Z. Zhan and S. Zhao, Sens. Actuators, B, 2015, 219, 50-56.

48 L. Yan, Y. Yang, C.-Q. Ma, X. Liu, H. Wang and B. Xu, Carbon, 2016, 109, 598-607.

49 H. Shen, W. Cao, N. T. Shewmon, C. Yang, L. S. Li and J. Xue, Nano Lett., 2015, 15, 1211-1216. 